\title{
Seeded self-modulated laser wakefield acceleration
}

\author{
N.E. Andreev, S. V. Kuznetsov, and A. A. Pogosova \\ Institute for High Energy Densities, Russian Academy of Sciences, Izhorskaya 13/19, Moscow 125412, Russia
}

L. C. Steinhauer

University of Washington, Redmond Plasma Physics Laboratory, Redmond, Washington 98052 USA

W. D. Kimura*

STI Optronics, Inc., Bellevue, Washington 98004 USA

(Received 13 January 2006; published 31 March 2006)

\begin{abstract}
A new approach to laser-wakefield acceleration (LWFA) has been analyzed. A seed electron beam bunch precedes the laser pulse into the plasma. This seed bunch initiates formation of plasma waves via a plasma wakefield acceleration mechanism. The amplitude of the plasma waves is subsequently amplified by the laser pulse via a self-modulated LWFA (SM-LWFA) process. This method enables the generation of strong wakefields even when the laser pulse by itself has characteristics that are insufficient for driving resonant LWFA or SM-LWFA. Another advantage is the wakefield formation begins at the seed bunch and does not start from noise as typically occurs in SM-LWFA. This feature may be helpful when the phase of the wakefield must be accurately controlled, for example, when staging multiple LWFA devices in series.
\end{abstract}

DOI: 10.1103/PhysRevSTAB.9.031303

PACS numbers: 41.75.Jv, 52.38.Kd, 42.55.Lt

\section{INTRODUCTION}

Laser-wakefield acceleration (LWFA) has demonstrated very high acceleration gradients in numerous experiments [1]. LWFA is typically initiated by sending a few tens of terawatt (TW) laser pulse into a plasma to create longitudinal plasma waves or wakefields [2]. These waves travel at near the speed of light and can accelerate electrons trapped within their potential well. When the laser pulse length $\tau_{L}$ is less than of order $\lambda_{p} / 2 c$, where $\lambda_{p}$ is the plasma wavelength and $c$ is the speed of light, this wakefield generation is referred to as resonant LWFA.

In a variation of the LWFA method, called selfmodulated LWFA (SM-LWFA) [3], the laser pulse length is much longer than $\lambda_{p} / 2 c$, but the laser intensity is still very high. This permits the laser electric field to feed energy into the wakefield via forward Raman scattering and/or a self-modulation instability. This enhances the wakefield formation process allowing much higher gradients to be produced compared to resonant LWFA. However, for wakefield amplitudes of interest, SMLWFA is a highly nonlinear process that typically starts from noise, so that the phase of the resulting wake is essentially uncontrolled.

Wakefield formation in a plasma is also possible by using an ultrashort electron beam ( $e$-beam) bunch rather than a laser pulse. This is referred to as plasma wakefield acceleration (PWFA) [4]. The formation mechanism in PWFA is analogous to resonant LWFA; hence, the resultant wakefields can have similar characteristics.

\footnotetext{
*Electronic address: wkimura@stioptronics.com
}

This paper presents the modeling analysis for a new method of laser-wakefield generation, which we call seeded SM-LWFA. (This concept was first introduced as "stimulated LWFA" [5]). It is essentially a hybrid of PWFA and SM-LWFA where an ultrashort $e$-beam bunch generates a wakefield in a plasma via PWFA. A laser pulse immediately follows the $e$-beam bunch and amplifies the wakefield via the SM-LWFA process.

The motivations for the development of this novel method are twofold. First, LWFA experiments are being conducted at the Brookhaven National Laboratory Accelerator Test Facility (BNL-ATF) that will use a TW $\mathrm{CO}_{2}$ laser to drive the acceleration process [6]. However, the $\mathrm{CO}_{2}$ laser pulse length is too long for resonant LWFA. Furthermore, conventional SM-LWFA is not feasible because simulations indicate at the present laser power levels that transverse wakefields tend to be generated rather than longitudinal ones. Seeded SM-LWFA circumvents these limitations by using an $e$-beam to initiate a longitudinal wakefield with amplitude much larger than the noise level, which the laser pulse can then amplify.

Second, the ultimate aim of the LWFA experiments at the ATF is to demonstrate efficient trapping and acceleration of electron microbunches while maintaining a narrow energy spread (i.e., monoenergetic). This is an important requirement for any practical laser-driven linear accelerator. The strategy being followed is the same one proved successful during the staged electron laser acceleration (STELLA) experiments [7]. STELLA demonstrated efficient trapping and monoenergetic acceleration using a twostage laser acceleration system based upon inverse free electron lasers (IFEL) [8]. The first IFEL stage modulated the $e$-beam energy thereby creating a train of micro- 
bunches. The second IFEL trapped and accelerated these microbunches. Inherent in this basic approach is the need to rephase the microbunches with the accelerating field in the second acceleration stage. This implies the need to control the phase of the accelerating field.

During resonant LWFA or PWFA, the phase of the generated wakefield should be closely correlated to a feature of the laser or $e$-beam pulse shape, respectively. (The former assumes the laser power is below the threshold for self-focusing of the laser beam in the plasma [9]). Hence, seeded SM-LWFA may also provide a means for generating wakefields whose phase is more controllable than in conventional SM-LWFA where the wakefield arises from noise. The ATF LWFA experiment, which is referred to as STELLA-LW where LW stands for laser wakefield, would be one of the first to investigate control of the wakefield phase in this manner.

Note that there is another potential method for controlling the phase and character of SM-LWFA by using a second low-intensity frequency-shifted laser pulse, which provides a seed for the main laser pulse self-modulation and does not rely on noise to generate the wakefields $[10,11]$.

The next section reviews changes made to an existing LWFA model to simulate the seeding process and the affects of the amplified wakefield on witness electrons that follow the seed $e$-beam bunch. Section III presents the model predictions assuming the ATF linac and $\mathrm{CO}_{2}$ laser characteristics. Conclusions follow in Sec. IV.

\section{MODIFICATIONS TO LWFA MODEL}

As explained, seeded SM-LWFA requires a seed $e$-beam bunch and laser pulse. A second $e$-beam bunch ("witness" bunch) follows the seed and laser pulses to provide electrons that interact with the amplified wakefield.

The ATF linac is capable of providing two $e$-beam bunches separated by a short time interval (e.g., 1020 ps) [12]. The first bunch serves as the seed and the second as the witness. The ATF can also send these dual bunches through a magnetic chicane such that the first bunch is compressed to less than $200 \mathrm{fs}$ while the duration of the second bunch remains largely unchanged. This compression of the first bunch is necessary for efficient seed wake generation. The length of the second witness bunch is less critical.

A model to simulate LWFA generation using a $\mathrm{CO}_{2}$ laser pulse was developed earlier in conjunction with analysis of another scheme called pseudoresonant LWFA [13]. This model is well suited for also simulating seeded SM-LWFA; however, it required two modifications. The first is to introduce a seed $e$-beam bunch whose wakes interact with the laser pulse propagating through the plasma. The second is to add the witness bunch. Because the formation of the wakefields and their amplification does not occur immediately after entering the plasma, it is important to include the effects of the plasma on the witness electrons before they reach the high-amplitude wakefields.

Equations (1)-(4) below are reproductions of Eqs. (4)(7) from [13] except for the addition of a new term $\mathbf{j}_{b}$ in Eq. (3) that represents the current density of the seed $e$-beam bunch.

$$
\begin{gathered}
\frac{\partial \mathbf{p}}{\partial t}=e \mathbf{E}-m c^{2} \nabla \gamma, \\
\frac{\partial n}{\partial t}+\nabla \cdot(n \mathbf{v})=0, \\
\frac{\partial \mathbf{E}}{\partial t}=-4 \pi e n \mathbf{v}+c \nabla \times \mathbf{B}-4 \pi \mathbf{j}_{b}, \\
\nabla \times \mathbf{E}=-\frac{1}{c} \frac{\partial \mathbf{B}}{\partial t},
\end{gathered}
$$

where $e$ is the electron charge; $\mathbf{E}$ and $\mathbf{B}$ are the electric field and magnetic flux in the plasma, respectively; $m$ is the mass of the electron; $\gamma=\left[1+(\mathbf{p} / m c)^{2}+|a|^{2} / 2\right]^{1 / 2} ; n$ is the plasma electron density; and $\mathbf{p}$ and $\mathbf{v}$ are the electron momentum and velocity, respectively. The dimensionless envelope amplitude $a$ of the laser pulse is related to the electric field of the laser pulse $\mathbf{E}_{0}$ by the expression

$$
e \mathbf{E}_{0} /\left(m \omega_{0} c\right)=\operatorname{Re}\left\{\mathbf{e}_{0} a \exp \left[-i \omega_{0} t+i k_{0} z\right]\right\},
$$

where $\omega_{0}$ and $k_{0}=\omega_{0} / c$ are the frequency and wave number of the laser radiation, respectively. Here $\mathbf{e}_{0}$ is the unit vector of the laser polarization, which is assumed to be linear. The quantity $a$ is assumed to vary slowly on the time and spatial scales $\omega_{0}^{-1}$ and $k_{0}^{-1}$, respectively.

Equations (1)-(4) describe the slowly varying motions and fields in the plasma. The seed $e$-beam is assumed to be relativistic with a current that is some given function of time and space, e.g., Gaussian.

As done in [13], we make a quasistatic approximation [14] by defining dimensionless coordinates moving with the laser pulse variables

$$
\xi=k_{p 0}(z-c t), \quad \zeta=k_{p 0} z, \quad \boldsymbol{\rho}=k_{p 0} \mathbf{r}_{\perp},
$$

where $k_{p 0}=\omega_{p 0} / c=\left(4 \pi e^{2} N_{0} / m\right)^{1 / 2} / c$ is the normalizing inverse space scale; $N_{0}$ is the unperturbed electron plasma density on the $e$-beam axis, $N_{0}=n_{0}(r=0)$; and $\mathbf{r}_{\perp}=\{x, y\}=r\{\cos \varphi, \sin \varphi\}$ is the radius vector in the radial direction $r$.

With the addition of the seed bunch, the linearized Eq. (16) of [13] for the wakefield potential variation $\delta \Phi$ becomes

$$
\begin{aligned}
& \left\{\left(\frac{\partial^{2}}{\partial \xi^{2}}+\nu_{0}\right)\left(\Delta_{\perp}-\nu_{0}\right)-\frac{\partial \ln \left[\nu_{0}(\rho)\right]}{\partial \rho} \frac{\partial^{2}}{\partial \xi^{2}} \frac{\partial}{\partial \rho}\right\} \delta \Phi \\
& \quad=\nu_{0}\left[\left(\Delta_{\perp}-\nu_{0}\right) \frac{|a|^{2}}{4}-N_{b}\right],
\end{aligned}
$$

where $\nu_{0}(\rho)=n_{0}(\rho) / N_{0}$ is the normalized electron back- 
ground density in the plasma channel; $N_{b}$ is the normalized

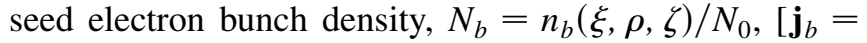
$\left.\left\{0,0, j_{b z}\right\}, j_{b z}=e n_{b}(\xi, \rho, \zeta) c\right]$; and $\Delta_{\perp}$ is the transverse part of the Laplacian operator. All other equations in the model have the same form as in [13].

To investigate the acceleration of relativistic electrons of the witness $e$-beam bunch in the wakefield we use the equations of motion in the form of [15]

$$
\begin{gathered}
\frac{d P_{z}}{d \tau}=F_{z}(\xi, \rho, \zeta), \\
\frac{d \mathbf{P}_{r}}{d \tau}=\mathbf{F}_{r}(\xi, \rho, \zeta), \\
\frac{d \xi}{d \tau}=\frac{P_{z}}{\sqrt{1+P_{z}^{2}+P_{r}^{2}}}-1, \\
\frac{d \boldsymbol{\rho}}{d \tau}=\frac{\mathbf{P}_{r}}{\sqrt{1+P_{z}^{2}+P_{r}^{2}}},
\end{gathered}
$$

where $P_{z}, \mathbf{P}_{r}=\left\{P_{x}, P_{y}\right\}$ are components of momentum, longitudinal and perpendicular to the axis $O Z$, of an accelerating electron normalized to $m c ; \tau=\omega_{p 0} t$; and $\zeta=\xi+$ $\tau$. The axial and radial components of the normalized force acting on the accelerating electron moving with velocity $c$ along the $O Z$ axis is a reasonable approximation for the relativistic witness $e$-beam bunch. These components can be expressed in terms of the normalized (to $m c^{2} / e$ ) wakefield potential $\Phi=1+\delta \Phi$ as follows:

$$
\begin{gathered}
F_{z} \equiv \frac{e E_{z}}{m c \omega_{p 0}}=\frac{\partial \Phi}{\partial \xi}, \\
F_{r} \equiv \frac{e E_{r}}{m c \omega_{p 0}}-\frac{e B_{\varphi}}{m c \omega_{p 0}}=\frac{\partial \Phi}{\partial \rho},
\end{gathered}
$$

where $\mathbf{F}_{r}=\left\{F_{x}, F_{y}\right\}=F_{r}\{\cos \varphi, \sin \varphi\}$.

In the simulations that follow we make the simplifying assumption of an infinite uniform plasma density in all directions. As a result the laser beam will exhibit modest diffractive spreading over the short plasma lengths modeled since the propagation distance is less than the laser beam Rayleigh range. In actual experiments [6], a capillary discharge could be used to guide the laser beam over longer distances.

\section{MODEL PREDICTIONS FOR SEEDED SM- LWFA}

The conventions for describing the $e$-beam and laser beam are as follows. The laser beam amplitude $a$ is given by

$$
a(r, z=0, t)=a_{0} \exp \left[-\frac{r^{2}}{w_{0}^{2}}-\frac{\left(t-t_{a}\right)^{2}}{\tau_{\mathrm{L}}^{2}}\right],
$$

and the $e$-beam density distribution is described as

$$
n_{b}(r, z=0, t)=n_{b 0} \exp \left[-\frac{r^{2}}{2 \sigma_{e}^{2}}-\frac{\left(t-t_{b}\right)^{2}}{2 \tau_{e}^{2}}\right],
$$

where $t_{a}$ and $t_{b}$ represent the delay times between the various pulses; $\sigma_{e} \equiv \sigma_{e \text {-seed }}, t_{b} \equiv t_{b \text {-seed }}$, and $\tau_{e} \equiv$ $\tau_{e \text {-seed }}$ for the seed $e$-beam bunch; and $\sigma_{e} \equiv \sigma_{e \text {-witness }}$, $t_{b} \equiv t_{b \text {-witness }}$, and $\tau_{e} \equiv \tau_{e \text {-witness }}$ for the witness $e$-beam bunch. These seemingly mixed conventions were adopted because of their prevalent usage within the laser (for $a$ ) and accelerator (for $n_{b}$ ) communities. For example, $w_{0}$ is the usual waist of the laser beam focus, whereas $\sigma_{e}$ is the rms size of the $e$-beam.

Table I lists the parameter values used in the model, which were chosen to simulate the approximate anticipated conditions at the ATF when utilizing the magnetic chicane to compress the seed $e$-beam bunch. As mentioned, the assumption is made that the plasma density is uniform over the entire plasma length; in reality, it may vary longitudinally when the capillary is short, e.g., 3-4 mm.

One important parameter to note is the plasma density, which is approaching $10^{17} \mathrm{~cm}^{-3}$. This is nearly $10 \times$ higher than the density required for pseudoresonant LWFA [13]. It can be higher because the compressed seed $e$-beam bunch length, of order $\sim 100-200 \mathrm{fs}$, is short enough for efficient seed wake generation at this higher density.

In the model predictions that follow, two cases are examined where the parameters are essentially the same except for differences in the temporal position of the seed bunch $t_{d}=t_{b \text {-seed }}-t_{a}$ within the laser pulse envelope. The delay time $t_{d}$ is measured between the peaks of the laser pulse and the seed $e$-beam bunch at the capillary entrance. This shows the sensitivity of the seeding process to this time delay.

\section{A. Time delay between laser and seed pulses $t_{d}=$ $2.97 \mathrm{ps}$}

Figures 1-5 show the results for the case when $t_{d}=$ 2.97 ps. In Fig. 1, the peak of the wakefield potential and the laser field maximum on axis are shown. The maximum of the laser field at the plasma boundary is chosen to be at $\xi=\xi_{a}=200$. The seed $e$-beam bunch propagates ahead of the laser pulse at $\xi_{b}=250$, which corresponds to a time delay between the laser pulse and seed $e$-beam bunch of $t_{d}=2.97 \mathrm{ps}$. This can be seen more clearly in Fig. 2, where the dashed line represents the laser pulse envelope at the plasma entrance $(z=0)$. The oscillating (red) line just below is the envelope after the pulse propagates in the plasma a distance $z=2.62 \mathrm{~mm}$. It is slightly lower because of some defocusing of the laser beam caused by diffraction. (For the conditions simulated, depletion of laser pulse energy is negligible.) More importantly, it shows the modulation characteristics of a strong interaction with the seed wake. This results in a growth of the 
TABLE I. Laser and plasma parameters used in seeded SM-LWFA simulations.

\begin{tabular}{|c|c|}
\hline Parameter & Value \\
\hline Laser wavelength, $\lambda_{L}$ & $10.6 \mu \mathrm{m}$ \\
\hline Laser pulse duration, $\tau_{L}{ }^{\mathrm{a}}$ & $8.44 \mathrm{ps}$ \\
\hline Laser peak power, $P_{L}$ & $0.5 \mathrm{TW}$ \\
\hline Laser pulse energy, $E_{L}$ & $5.3 \mathrm{~J}$ \\
\hline Laser beam focus radius, $w_{0}$ & $111 \mu \mathrm{m}$ \\
\hline Laser beam Rayleigh range, $z_{R}$ & $3.64 \mathrm{~mm}$ \\
\hline Normalized laser field strength, $a_{0}$ & 0.462 \\
\hline Plasma length ${ }^{\mathrm{b}}$ & $2-3 \mathrm{~mm}$ \\
\hline Plasma density on axis, $n_{0}{ }^{\mathrm{c}}$ & $0.89 \times 10^{17} \mathrm{~cm}^{-3}$ \\
\hline$P_{L} / P_{\text {crit }}$ (for self-focusing) & 0.265 \\
\hline Time delay between peak of laser pulse and peak of seed $e$-beam bunch, $t_{d}$ & 2.97 or $8.91 \mathrm{ps}$ \\
\hline$E$-beam energy (seed and witness), $E_{\text {inj }}$ & $64 \mathrm{MeV}$ \\
\hline Seed $e$-beam intrinsic energy spread [\%] & $\ll 1 \% \mathrm{~d}$ \\
\hline Seed $e$-beam bunch charge & $199 \mathrm{pC}$ \\
\hline Seed $e$-beam bunch length, $\tau_{e \text {-seed }}$ & $118 \mathrm{fs}$ \\
\hline Seed $e$-beam focus size at capillary, $\sigma_{e \text {-seed }}$ & $50 \mu \mathrm{m}$ \\
\hline Witness $e$-beam intrinsic energy spread [\%] & 0.05 \\
\hline Witness $e$-beam bunch charge & $\ll 500 \mathrm{pC}^{\mathrm{e}}$ \\
\hline Witness $e$-beam bunch length, $\tau_{e \text {-witness }}$ & $1.23 \mathrm{ps}$ \\
\hline Witness $e$-beam focus size at capillary, $\sigma_{e \text {-witness }}$ & $20 \mu \mathrm{m}$ \\
\hline Time delay between seed and witness $e$-beam bunches, $\tau_{d}$ & $6-21 \mathrm{ps}$ \\
\hline
\end{tabular}

${ }^{a}$ The full-width-at-half-maximum pulse duration of the laser intensity is equal to $\tau_{\mathrm{FWHM}}=2 \sqrt{\ln 2} \tau_{\mathrm{L}}=9.93 \mathrm{ps}$.

${ }^{\mathrm{b}}$ The plasma length is assumed to be the same as the capillary length.

${ }^{c}$ The plasma density is assumed uniform over the entire plasma length.

${ }^{\mathrm{d}}$ The seed $e$-beam propagates with constant velocity determined by its energy without changing its shape, so the results do not depend on the initial energy spread.

${ }^{\mathrm{e}}$ Not critical assuming loading effects can be ignored.

wakefield potential as shown plotted to the right of the seed bunch in Fig. 2.

Note that the wakefield potential over the first $1.5 \mathrm{~mm}$ propagation distance (see Fig. 1) is not the pure amplitude of the plasma wave (which is responsible for accelerating the electrons), but also reflects the ponderomotive potential of the laser pulse.



FIG. 1. (Color) Model prediction for the maximum of laser field parameter $|a(r=0, z)|_{\max }$ and wakefield potential $\delta \Phi_{\max }(r=$ $0, z)$ as a function of distance along the plasma for $t_{d}=2.97 \mathrm{ps}$.
In Fig. 3, we see that the wakefields are predominately longitudinal after a propagation distance of $2.62 \mathrm{~mm}$. They remain longitudinal even for distances over $5 \mathrm{~mm}$ (not shown). The radial extent of the strongest wakefields ex-



FIG. 2. (Color) Model prediction for laser field parameter $|a(r=0)|$ and wakefield potential $\delta \Phi(r=0)$ as a function of time for $z=2.62 \mathrm{~mm}$ and $t_{d}=2.97 \mathrm{ps}$. Also plotted are the seed and witness $e$-beam bunch positions for $\tau_{d}=12 \mathrm{ps}$ and $|a(r=0, z=0)|$. 


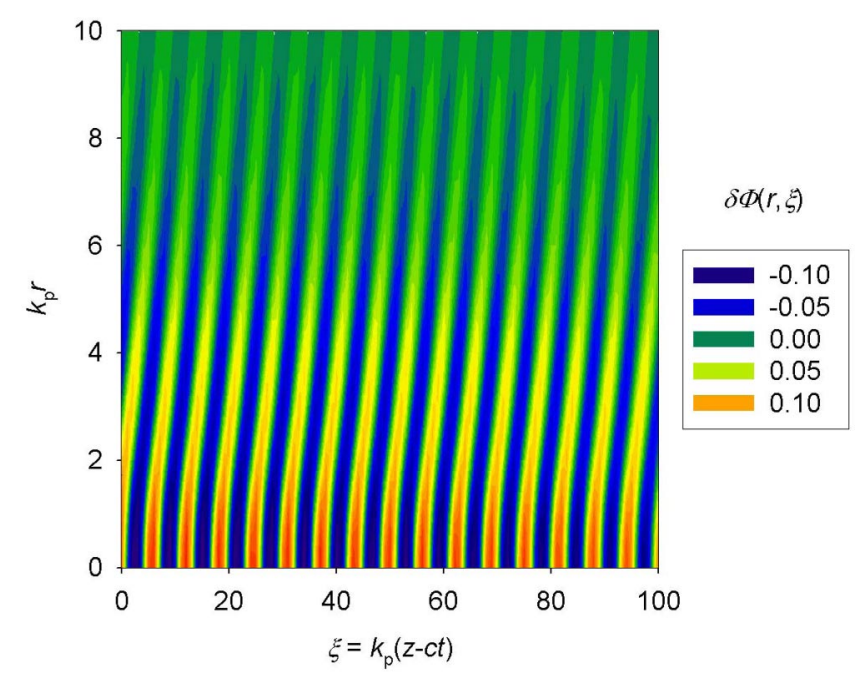

FIG. 3. (Color) Model prediction for wakefield potential distribution $\delta \Phi(r, \xi)$ plotted after a propagation distance of $2.62 \mathrm{~mm}$ for $t_{d}=2.97 \mathrm{ps}$.

tends to approximately $k_{p} r=3$. For a plasma density of $8.9 \times 10^{16} \mathrm{~cm}^{-3}$, the plasma wavelength $\lambda_{p}=112 \mu \mathrm{m}$ and $k_{p}=2 \pi / \lambda_{p}=561 \mathrm{~cm}^{-1}$. Hence, $k_{p} r=3$ corresponds to a radial distance of $53 \mu \mathrm{m}$. Thus, the witness $e$-beam bunch rms radius should be less than this distance.

These figures demonstrate effective wakefield generation after propagation over the first $2 \mathrm{~mm}$ (representing the initial stage of self-modulation development). Moreover, the wakefields generated are primarily longitudinal rather than transverse. This overcomes the problem that is predicted to appear if the ATF $\mathrm{CO}_{2}$ laser pulse alone is used to generate wakefields via SM-LWFA.

Figure 2 also illustrates a typical time separation $\tau_{d}$ between the seed and witness $e$-beam bunches, and their

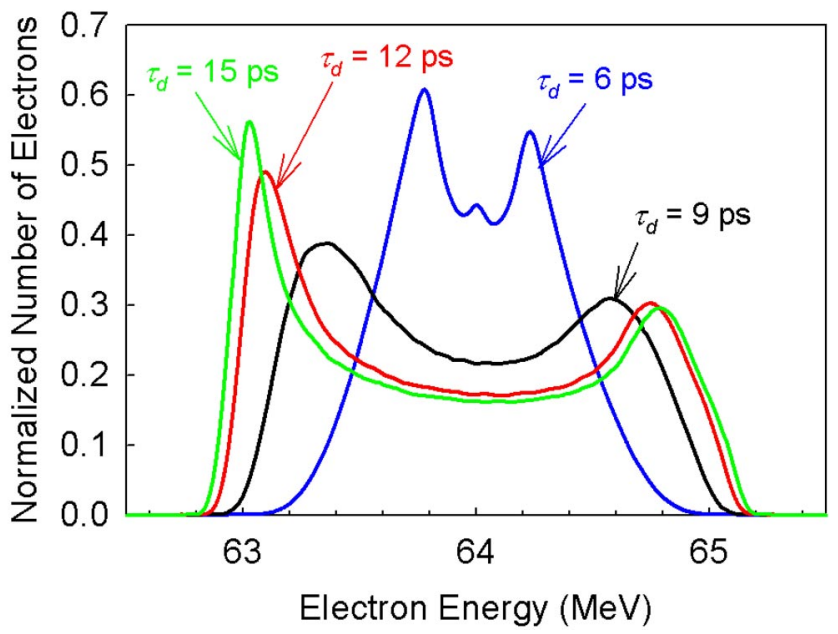

FIG. 4. (Color) Model prediction for energy spectrum of witness bunch for $t_{d}=2.97 \mathrm{ps}$ and different time delays between the seed and witness $e$-beam bunches for an acceleration length $L_{\text {acc }}=2 \mathrm{~mm}$. temporal positions relative to the laser pulse. In this particular case, $\tau_{d}=12 \mathrm{ps}$.

Figure 4 presents the predicted energy spectrum of the witness bunch for $\sigma_{e \text {-witness }}=20 \mu \mathrm{m}, \tau_{e \text {-witness }}=1.23 \mathrm{ps}$, and an acceleration length $L_{\text {acc }}=2 \mathrm{~mm}$. Plotted are the spectrums for different $\tau_{d}$ between the seed and witness $e$-beam bunches. The intrinsic energy spread of the injected witness $e$-beam bunch is neglected in this figure. (Other model runs show that a small intrinsic energy spread of the witness $e$-beam, which is much smaller than the final energy spectrum, does not change the energy modulation characteristics of the witness bunch.) A double-peak energy spectrum is observed, which is evidence of sinusoidal energy modulation [16]. This peak modulation appears to reach a steady-state maximum value of $\approx \pm 1 \mathrm{MeV}$ for time separations greater than $\tau_{d} \sim 10 \mathrm{ps}$. This implies the time delay between the seed and witness $e$-beam bunches is not critical and delays of $\tau_{d} \approx 10-20$ ps are acceptable.

An acceleration distance of $2 \mathrm{~mm}$ is comparable to the dephasing length calculated by using the average phase velocity of the wakefield at $z \approx 2 \mathrm{~mm}$, i.e., $L_{d p h}=$ $\lambda_{p} \bar{\gamma}_{p h}^{2} \cong 1.8 \mathrm{~mm}$ with $\bar{\gamma}_{p h} \cong 4$, which is derived from analysis of the wakefield phase obtained in separate calculations. (Note the relativistic parameter associated with the group velocity of the laser pulse is $\gamma_{g}=\omega_{0} / \omega_{p} \cong 10.6$.) This explains why the spectra in Fig. 4 are more symmetrical than those that will be shown later for $L_{\text {acc }}=$ $3 \mathrm{~mm}$.

The spectrum for the shortest time separation $\left(\tau_{d}=\right.$ $6 \mathrm{ps}$ ) displays a small peak at the mean $e$-beam energy (i.e., $64 \mathrm{MeV}$ ). This is caused by a small number of witness electrons that are located on the low amplitude wakefield near the seed $e$-beam bunch position. This peak will also be clearly seen later in Fig. 5.

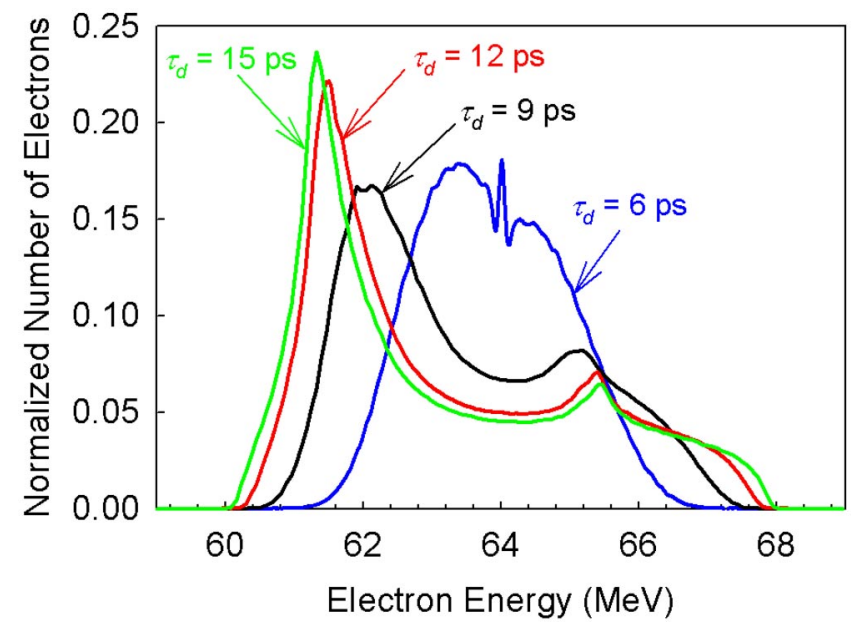

FIG. 5. (Color) Model prediction for energy spectrum of witness bunch for $t_{d}=2.97 \mathrm{ps}$ and different time delays between the seed and witness $e$-beam bunches for an acceleration length $L_{\text {acc }}=3 \mathrm{~mm}$. 
Increasing the acceleration length by $50 \%$ to $L_{\mathrm{acc}}=$ $3 \mathrm{~mm}$, the predicted energy spectra are shown in Fig. 5 . The maximum amount of modulation has increased by $\sim 400 \%$ to nearly $\pm 4 \mathrm{MeV}$ and the spectra have become considerably asymmetric in shape. The more pronounced asymmetry of the spectrum (in comparison with Fig. 4) is a consequence of the acceleration distance, $L_{\text {acc }}=3 \mathrm{~mm}$, exceeding the dephasing length, $L_{\mathrm{dph}} \cong 1.8 \mathrm{~mm}$, which leads to deceleration of a substantial part of the electrons in the witness bunch.

\section{B. Time delay between laser and seed pulses $t_{d}=8.91 \mathrm{ps}$}

Figure 6 shows the relationship between the various pulses and fields when the time delay between the laser and seed pulse is increased to $t_{d}=8.91 \mathrm{ps}$. Comparing Fig. 6 with Fig. 2, this is achieved by having the seed $e$-beam bunch arrive earlier with respect to the laser pulse. The witness $e$-beam bunch is essentially in the same time location with respect to the laser pulse. Moving the seed bunch earlier allows the wakefields to begin growing earlier within the laser pulse thereby resulting in higher amplitude of the wakefield potential, cf. wakefield potential plots in Figs. 2 and 6.

Figure 7 shows the energy spectrum for different time separations between the seed and witness $e$-beam bunches for a witness $e$-beam bunch with an intrinsic energy spread of $\sigma_{\mathrm{E}}=0.05 \% E_{\mathrm{inj}}$ and an acceleration length $L_{\mathrm{acc}}=$ $3 \mathrm{~mm}$. The maximum energy modulation has now increased to over $\pm 6 \mathrm{MeV}$. This corresponds to an acceleration gradient of $2 \mathrm{GeV} / \mathrm{m}$.

There is also a larger concentration of decelerated electrons at approximately $60.5 \mathrm{MeV}$ due to increased decel-

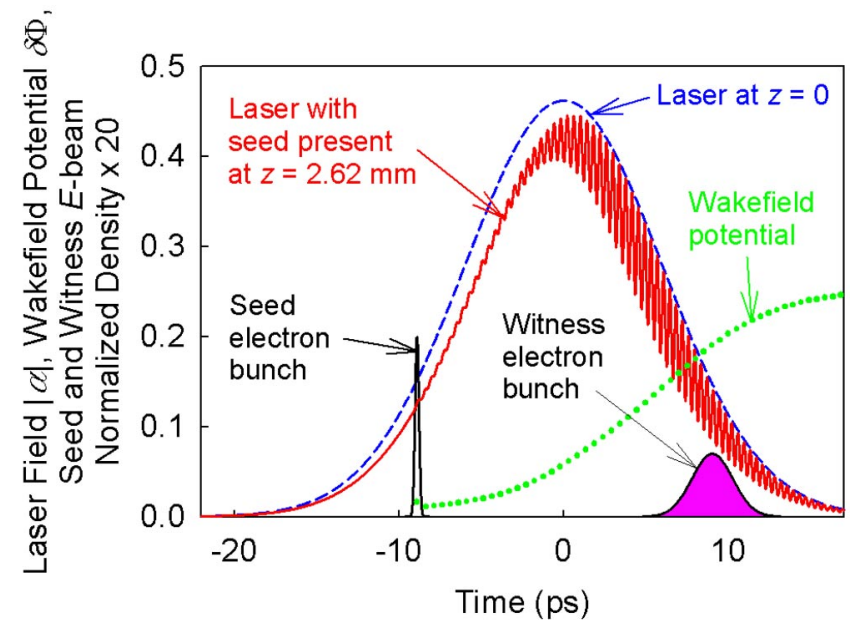

FIG. 6. (Color) Model prediction for laser field parameter $|a(r=0)|$ and wakefield potential $\delta \Phi(r=0)$ as a function of time for $t_{d}=8.91 \mathrm{ps}$. Also plotted are the seed and witness $e$-beam bunch positions for $\tau_{d}=17 \mathrm{ps}$ and $|a(r=0, z=0)|$.



FIG. 7. (Color) Model prediction for energy spectrum of witness bunch for $t_{d}=8.91 \mathrm{ps}$ and different time delays between the seed and witness $e$-beam bunches for an acceleration length $L_{\text {acc }}=3 \mathrm{~mm}$.

erating forces (as compared with Figs. 4 and 5) for the main portion of electrons in the witness $e$-beam bunch because the acceleration length exceeds the dephasing length.

Moreover, in our simulations the witness bunch length $(\sim 1000 \mu \mathrm{m})$ is much longer than the plasma wavelength $(\sim 100 \mu \mathrm{m})$, which results in the energy modulation seen in the figures. Efficient, monoenergetic acceleration is possible if the witness bunch length can made of order $1 / 10$ the plasma wavelength. This should be achievable using a technique similar to the STELLA experiment [16], where the witness electrons are energy modulated in a short-length, seeded SM-LWFA device and allowed to form a train of microbunches. These microbunches can be subsequently sent into a second LWFA device and rephrased with the plasma wave for maximum acceleration.

\section{CONCLUSION}

A novel method for generating wakefields has been analyzed, which combines PWFA and SM-LWFA. It provides several potential benefits: (1) It enables strong wakefield generation that is comparable to conventional SMLWFA, but where the laser pulse characteristics are insufficient to initiate the SM-LWFA process by using the laser pulse only. (2) The longitudinal structure of the wakefield is tied closely to the seed $e$-beam bunch, which may enable more precise control of the wakefield phase, thereby facilitating staging of LWFA devices. (3) For the conditions at the ATF, seeded SM-LWFA permits operation at considerably higher plasma densities than pseudoresonant LWFA, thereby easing the operational requirements of a capillary discharge.

The STELLA-LW experiment plans a proof-of-principle demonstration of seeded SM-LWFA in the near future [17] with the goal of observing the type of energy modulation 
predicted in this paper. Future experiments would build upon this proof-of-principle experiment to demonstrate microbunch formation, staging, and monoenergetic acceleration.

\section{ACKNOWLEDGMENTS}

This work was supported by the U.S. Department of Energy, Grant No. DE-FG02-04ER41294.

[1] See, for example, W.P. Leemans and E. Esarey, in Advanced Accelerator Concepts: Eighth Workshop, edited by W. Lawson, C. Bellamy, and D. Brosius, AIP Conf. Proc. No. 472 (American Institute of Physics, New York, 1999), p. 174.

[2] T. Tajima and J.M. Dawson, Phys. Rev. Lett. 43, 267 (1979); L. M. Gorbunov and V.I. Kirsanov, Sov. Phys. JETP 66, 290 (1987).

[3] N.E. Andreev, L. M. Gorbunov, V.I. Kirsanov, A. A. Pogosova, and R. R. Ramazashvili, Pis'ma Zh. Eksp. Teor. Fiz. 55, 551 (1992) [JETP Lett. 55, 571 (1992)].

[4] P. Chen, J. M. Dawson, R. W. Huff, and T. Katsouleas, Phys. Rev. Lett. 54, 693 (1985).

[5] L. C. Steinhauer, W. D. Kimura, and R. N. Agarwal, in Proceedings of International Conference on Lasers 2001, edited by V. J. Corcoran and T. A. Corcoran (STS Press, McLean, 2002), pp. 159-163.

[6] W. D. Kimura, N.E. Andreev, M. Babzien, I. Ben-Zvi, D. B. Cline, C.E. Dilley, S. C. Gottschalk, S. M. Hooker, K. P. Kusche, S. V. Kuznetsov, R. H. Pantell, I. V. Pavlishin, I. V. Pogorelsky, A. A. Pogosova, L.C. Steinhauer, A. Ting, V. Yakimenko, A. Zigler, and F. Zhou, IEEE Trans. Plasma Sci. 33, 3 (2005).
[7] W. D. Kimura, M. Babzien, I. Ben-Zvi, L. P. Campbell, D. B. Cline, C. E. Dilley, J. C. Gallardo, S. C. Gottschalk, K.P. Kusche, R.H. Pantell, I. V. Pogorelsky, D.C. Quimby, J. Skaritka, L. C. Steinhauer, V. Yakimenko, and F. Zhou, Phys. Rev. Lett. 92, 054801 (2004).

[8] R. B. Palmer, J. Appl. Phys. 43, 3014 (1972).

[9] P. Sprangle, E. Esarey, A. Ting, and G. Joyce, Appl. Phys. Lett. 53, 2146 (1988).

[10] N.E. Andreev, L. M. Gorbunov, and V.I. Kirsanov, Fiz. Plazmy 21, 872 (1995) [Plasma Phys. Rep. 21, 824 (1995)]; Phys. Plasmas 2, 2573 (1995).

[11] M. Fomyts'kyi, C. Chiu, M. Downer, and F. Grigsby, Phys. Plasmas 12, 023103 (2005).

[12] F. Zhou, D. Cline, M. Babzien, V. Yakimenko, and W. D. Kimura, in Proceedings of the Particle Accelerator Conference, Knoxville, TN, 2005 (IEEE, Piscataway, NJ, 2005), pp. 2312-2314 [IEEE Cat. No. 05CH37623C].

[13] N. E. Andreev, S. V. Kuznetsov, A. A. Pogosova, L. C. Steinhauer, and W.D. Kimura, Phys. Rev. ST Accel. Beams 6, 041301 (2003).

[14] P. Sprangle, E. Esarey, and A. Ting, Phys. Rev. Lett. 64, 2011 (1990).

[15] N.E. Andreev, S. V. Kuznetsov, I. V. Pogorelsky, Phys. Rev. ST Accel. Beams 3, 021301 (2000).

[16] W.D. Kimura, L.P. Campbell, C.E. Dilley, S.C. Gottschalk, D. C. Quimby, M. Babzien, I. Ben-Zvi, J.C. Gallardo, K. P. Kusche, I. V. Pogorelsky, J. Skaritka, V. Yakimenko, D. B. Cline, F. Zhou, L. C. Steinhauer, and R. H. Pantell, Phys. Rev. ST Accel. Beams 7, 091301 (2004).

[17] W.D. Kimura, N.E. Andreev, M. Babzien, I. Ben-Zvi, D. B. Cline, C. E. Dilley, S. C. Gottschalk, S. M. Hooker, K. P. Kusche, S. V. Kuznetsov, I. V. Pavlishin, I. V. Pogorelsky, A. A. Pogosova, L. C. Steinhauer, A. Ting, V. Yakimenko, A. Zigler, and F. Zhou, Phil. Trans. R. Soc. A 364, 611 (2006). 